\title{
Role of Pax4 in Pdx1-VP16-mediated liver-to-endocrine pancreas transdifferentiation
}

\author{
Dong-Qi Tang ${ }^{1}$, Li-Zhen Cao ${ }^{1}$, Wayne $\mathrm{Chou}^{2}$, Lu Shun ${ }^{1}$, Christine Farag ${ }^{1}$, \\ Mark A Atkinson ${ }^{1}$, Shi-Wu Li ${ }^{1}$, Lung-Ji Chang ${ }^{2}$ and Li-Jun Yang ${ }^{1}$ \\ ${ }^{1}$ Department of Pathology, Immunology, and Laboratory Medicine, University of Florida College of Medicine, \\ Gainesville, FL, USA and ${ }^{2}$ Department of Molecular Genetics and Microbiology, University of Florida College \\ of Medicine, Gainesville, FL, USA
}

\begin{abstract}
Although Pdx1-VP16 expression induces hepatic cell transdifferentiation into pancreatic precursor cells (WB-1), these incompletely reprogrammed cells fail to become glucose-sensitive insulin-producing cells in the absence of the activation of late-stage pancreatic transcription factors. As Pax4 promotes late-stage $\beta$-cell differentiation and maturation, we generated lentiviral vector (LV) containing mouse Pax4 gene and developed two hepatic cell lines expressing Pax4 in the absence (WB-2 cells) or presence (WB-1A cells) of Pdx1-VP16, via LV-mediated gene transfer. Functional Pax4 protein expression in WB-2 and WB-1A cells was confirmed by electrophoretic mobility shift assay and Pdx1-VP16 protein expression in WB-1 and WB-1A cells was confirmed by Western blotting. Activation of Pax4 resulted in the expression of the late-stage transcription factors, including Pax6, Isl-1, and MafA, and generated a gene expression profile for WB-1A cells similar to that of functional rat insulinoma INS-1 cells. Insulin abundance in WB-1A cells was demonstrated by immunostaining. WB-1A cells exhibited glucose-responsive insulin release in vitro, and caused a rapid reversal of hyperglycemia following cell transplantation into streptozotocin-induced diabetic mice. Intraperitoneal glucose tolerance test showed a normal glucose response in WB-1, and WB-1A transplanted mice similar to that of normal mice. Removal of transplanted WB-1A cells resulted in a return of hyperglycemia, confirming that they were responsible for the observed normoglycemia. The explanted WB-1A cells exhibited strong insulin staining comparable to native islet $\beta$-cells. These studies indicate that activation of Pax4 in Pdx1-VP16-expressing cells reprograms pancreatic precursor-like WB-1 cells into glucose-responsive, more mature insulin-producing cells.
\end{abstract}

Laboratory Investigation (2006) 86, 829-841. doi:10.1038/labinvest.3700434; published online 29 May 2006

Keywords: transdiiferetiation; WB cells; Pdx1-VP16; Pax4; insulin-producing cells; type 1 diabetes

Despite the vital role of insulin replacement therapy, patients with type 1 diabetes (T1D) suffer many long-term complications associated with widely fluctuating blood glucose levels. The seemingly ideal prospect of islet cell transplantation is limited by the availability of sufficient transplant tissue as well as inherent risks of infection and/or rejection. Investigators are therefore actively seeking ways to generate functional insulin-producing cells to restore and maintain normoglycemia in vivo. As liver and pancreas are derived from a common embryonic bipotential precursor cell, ${ }^{1}$ liver-to-endocrine pan-

Correspondence: Dr L-J Yang, MD, Department of Pathology, Immunology, and Laboratory Medicine, University of Florida College of Medicine, 1600 SW Archer Road, PO Box 100275, Gainesville, FL 32610-0275, USA.

E-mail: yanglj@pathology.ufl.edu

Received 28 February 2006; revised 11 April 2006; accepted 15 April 2006; published online 29 May 2006 creas transdifferentiation offers an attractive strategy for generating $\beta$-cell surrogates. Pancreas-to-liver transdifferentiation has already been demonstrated both in vitro and in vivo by changing the microenvironment and/or activating transcription factor (TF) gene expression. ${ }^{2-5}$ Ectopic and transient expression of the pancreatic duodenal homeobox-1 $(P d x 1)$ gene, itself widely regarded as a master transcriptional regulator of pancreas development, transdifferentiates mouse liver cells into pancreatic endocrine and exocrine cells, ${ }^{5-8}$ and imparts a subsequent ability to reduce hyperglycemia in diabetic mice in vivo. ${ }^{6-8}$ However, hepatocyte conversion into endocrine pancreatic cells solely by ectopic $P d x 1$ expression is incomplete and nonselective ${ }^{5-8}$ leading to severe hepatitis due to such exocrine by-products as amylase and trypsin. ${ }^{8}$ The results of partial reprogramming underscore the need to engage other developmental cues encoded within the transcriptional factor cascade. 
To probe in vitro liver-to-endocrine pancreas transdifferentiation, we previously generated the WB-1 cell line expressing Pdx1-VP16 protein. $^{9}$ The Pdx1-VP16 fusion protein consists of mouse Pdx1 and the C-terminal activation domain (80aa) of the Herpes simplex viral protein VP16, thereby creating a super-active form of $\mathrm{Pdx} 1 .^{5}$ In that study, we demonstrated that $P d x 1-V P 16$ expression resulted in the transdifferentiation of hepatic WB cells into glucose-insensitive, pancreatic endocrine-precursor cells that express many genes related to early $\beta$-cell development, but exhibit no evidence of pancreatic exocrine differentiation. ${ }^{9}$ While not becoming fully functional pancreatic $\beta$-like cells, these precursor cells can mature into insulin-producing cells when provided with other cues, such as in vivo hyperglycemia or in vitro long-term culture at high glucose concentration. $^{9}$

We also observed that the late-stage transcription factor Pax4, Pax6, Isl-1 and MafA genes known to promote pancreatic endocrine $\beta$-cell differentiation and maturation were not expressed in the newly generated WB-1 cells. Pax4 is thought to be positioned upstream of those late-stage transcription factors $^{10}$ that are silent in WB-1 cells, but became active in WB-1 cells following in vivo further differentiation and maturation. ${ }^{9}$ Moreover, the Pax4 gene is expressed in the early pancreas, but is later restricted to $\beta$-cells. It has been proposed that the early expression of Pax4 in a subset of endocrine progenitors is essential for the differentiation of the beta and delta cell lineages. ${ }^{11}$ Inactivation of Pax4 by homologous recombination results in the absence of mature insulin- and somatostatinproducing cells (beta and delta, respectively) in the pancreas of Pax4 homozygous mutant mice, but glucagon-producing alpha cells are present in considerably higher numbers. ${ }^{11}$ However, the earliest insulin-producing precursor cells can be detected at embryonic day $8.5-9,{ }^{12}$ indicating that Pax4 expression is not absolutely necessary for the generation of $\beta$-cell precursors, but is critical for the proliferation and/or survival of these cells. ${ }^{11}$ Additionally, a recent work shows that expression of Pax4 promotes $\beta$-cell replication and survival in human and rat islets by modulating apoptosis through Bcl-xL expression and proliferation via C-myc levels. ${ }^{13}$

As Pax4 plays such a pivotal role in late-stage $\beta$-cell differentiation, proliferation, maturation, ${ }^{11}$ as well as survival, ${ }^{13}$ we sought to determine whether lentivirus-mediated Pax4 expression in Pdx1-VP16expressing WB-1 cells could promote a more completely differentiated phenotype with $\beta$-cell properties. Our results demonstrate that co-expression of Pax4 in Pdx1-VP16-expressing hepatic WB cells generated glucose-responsive insulin-producing cells, capable of inducing normoglycemia in diabetic mice more rapidly than cells expressing Pdx1-VP16 alone.

\section{Materials and methods}

\section{Plasmid Constructs}

Lentiviral vector (LV) containing Pax4 or GFP genes (Stratagene La Jolla, CA, USA) was constructed by inserting the entire mouse Pax4 coding sequence (gift of Michael German) ${ }^{14}$ or GFP into the pTYF vector cassette under control of the elongation factor$1 \alpha(\mathrm{eEF}-1 \alpha)$ promoter. Lentivirus was produced and the titer determined as previously described. ${ }^{15,16}$

\section{Transduced Cell Lines}

Rat liver epithelial WB cells representing the cultured counterpart of liver stem-like cells ${ }^{17,18}$ were provided by WB Coleman (University of North Carolina). WB-1 cells (expressing both Pdx1-VP16 and RIP-GFP genes) was previously generated as described in Cao et al. ${ }^{9}$ WB-2 cells (expressing Pax4 alone) were generated by transducing WB cells with LV-Pax4 vector. WB-1A cells (expressing both $P d x 1$ $V P 16$ and Pax4 genes) were generated by transducing WB-1 cells at passage 10 with LV-Pax4. Cell transduction by $\mathrm{LV}$ was conducted as previously described. ${ }^{15,16}$ Transduction efficiency was determined by transducing WB cells with $L V-G F P$ at a multiplicity of infection of 20.

\section{Culture Conditions}

WB-derived cell lines were maintained in RPMI 1640 medium supplemented with $10 \%$ FCS and $11.1 \mathrm{mM}$ D-glucose. The rat insulinoma INS-1 cell line (clone 832/13) (gift of Christopher B Newgard) has been maintained continuously for almost 2 years in RPMI 1640 medium and served as a positive control for rat $\beta$-cell gene expression, insulin and Pdx1 protein expression. ${ }^{19}$

\section{RT-PCR}

Total RNA was prepared from WB-derived cell lines at passages between 10 and 12 using TRI-reagent, and gene expression determined by RT-PCR. ${ }^{9,20}$ The forward and reverse PCR primers (see Supplementary Table 1 for exact sequences employed) were designed to be located in different exon(s).

\section{ELISA}

Insulin content in cell lysates and insulin release into the culture medium from the WB, WB-1, WB$1 \mathrm{~A}$, and WB-2 cells were preformed in triplicate as described elsewhere. ${ }^{9}$

\section{Western Blotting}

Pdx1 and Pdx1-VP16 proteins were detected according to our previously published methods. ${ }^{9,21}$ In brief, cell lysates ( $50 \mu \mathrm{g} / \mathrm{lane})$ were separated by SDS- 
PAGE using 12\% Tris-HCl gels (Bio-Rad). Proteins were transferred and blotted with rabbit anti-Pdx1 serum (1:5000), or anti-VP16 (1:200, BD Pharmagen), followed by HRP-conjugated secondary anti-rabbit polyclonal antibody $(1: 20,000)$, and visualized by enhanced chemiluminescence.

\section{Electrophoretic Mobility Shift Assays (EMSA)}

PAX4 binding sequence was selected based on published sequence of rat insulin I C2 element. ${ }^{14}$ The oligonucleotide probes and their locations in relation to the transcriptional start site for the gene are as shown (top strand): rat insulin I C2 element (rInsIC2), bp -328 to $-304, \quad 5^{\prime}$-CTGGGAAATGAGGTGGAAAATGCTC- $3^{\prime}$. The sense strand was labeled with the Biotin $5^{\prime}$ end DNA Labeling Kit (Pierce) and hybridized for $4 \mathrm{~h}$ at room temperature to form duplexes containing the binding sites for Pax4. Gel shift assays were performed using the Pierce LightShift Chemiluminescent EMSA Kit (Pierce) with $20 \mathrm{fmol}$ duplex per binding reaction. The source of the Pax4 protein was nuclear extracts prepared using Nuclear Extract kit (Active Motif) $(20 \mu \mathrm{g}$ nuclear protein per reaction). Due to no commercial Pax4 antibody available, we produced the His-tag purified recombinant Pax4-V5 protein and this protein $(0.5 \mu \mathrm{g})$ was used as positive control. Competition reactions containing a 200 -fold molar excess of unlabeled duplex of rInsIC2 were performed to illustrate the specificity of the protein:DNA interactions. Biotin-EBNA and unlabeled EBNA (Pierce, provided in the kit) were used as positive assay controls.

\section{Immunofluorescence}

Slides were prepared from cultured WB, WB-1, WB1A, WB-2 and INS-1 cell lines, fixed with methanol for $10 \mathrm{~min}$, and incubated with antibodies directed against insulin $(1: 500)$ or glucagon $(1: 100) .{ }^{22}$ DAPI was used to highlight cell nuclei. Mouse pancreas tissue and INS-1 cells served as positive controls for insulin and glucagon staining.

\section{Cell Transplantation}

Male NOD-scid mice (8-10 weeks old) were made hyperglycemic by multiple intraperitoneal injections of streptozotocin (Stz) at $50 \mu \mathrm{g} / \mathrm{g}$ body weight daily for 5 days, as previously described., ${ }^{9,22}$ When blood glucose levels reached $>350 \mathrm{mg} / \mathrm{dl}$, mice were transplanted with WB-1, WB-1A WB-2, or WB-GFP cells $\left(1 \times 10^{6}\right.$ cells/mouse $)$ into the left renal capsular space. The blood glucose levels were monitored routinely at 1600 in non-fasting conditions with no restriction to access to food and water. Transplanted cells were removed by left nephrectomy approximately day -40 posttransplantation in order to assess metabolic activity and morphologic characteristics. The control hyper- glycemic mice were terminated approximately day -40 post-transplantation. Intraperitoneal glucose tolerance test (IPGTT) was performed as previously described. ${ }^{21,22}$

\section{Histology and Immunohistochemistry}

The explanted tissues containing implanted WB, WB2, WB-1, and WB-1A cells from transplanted mice were fixed and embedded in paraffin, and sections were then stained with hematoxylin and eosin (H\&E). Sections were incubated with anti-insulin antibodies (1:500, Dako) as previously published. ${ }^{9}$

\section{Statistical Analysis}

The statistical significance of our experimental findings was analyzed by using an independent sample $t$-test, using a $P$-value of $<0.05$ for the data to be considered significant.

\section{Results}

Generation of WB-derived cell lines. We previously demonstrated that $P d x 1-V P 16$ expression in hepatic stem-like WB cells results in their transdifferentiation into $\beta$-cell precursor-like cells. ${ }^{9}$ The latter cells exhibit the capacity to mature into functional insulin-producing cells upon exposure in vivo to a diabetic hyperglycemic microenvironment. However, the newly generated WB-1 cells did not express Pax4, Pax6, Isl-1, and MafA genes normally appearing at late-stages of $\beta$-cell development. They also fail to release insulin in response to in vitro glucose stimulation. ${ }^{9}$ To study the role of Pax4 in the Pdx1VP16-mediated $\beta$-cell transdifferentiation, we first generated several WB-derived cell lines that express Pdx1-VP16 (WB-1), Pdx1-VP16/Pax 4 (WB-1A), Pax4 (WB-2), or GFP (WB) genes as outlined in Figure 1a and described in Materials and methods. Activation of Pax4 in WB-1 cells allowed for studies the role of Pax4 in Pdx1-VP16-mediated transdifferentiation of liver stem cell into insulin-producing cells; while introduction of Pax4 into WB cells allowed us to determine a role for Pax4 alone in the transdifferentiation. WB cells transduced with $L V$ GFP served as controls for $\mathrm{LV}$ vector and for evaluation of transduction efficiency. As demonstrated by counting GFP-positive WB cells $48 \mathrm{~h}$ after $L V$-GFP transduction (MOI of 20), the transduction efficiency was nearly $100 \%$ (Figure $1 \mathrm{~b}$ ), with no further selection of positive single-cell clones required for the purpose of our intended studies.

\section{Pdx1 and Pdx1-VP16 Protein Expression}

To confirm that the cell lines expressed Pdx1 and its fusion protein Pdx1-VP16, the actual proteins were detected by Western blotting with anti-Pdx1 (Figure 2a) and anti-VP16 (Figure 2b). As expected, both 


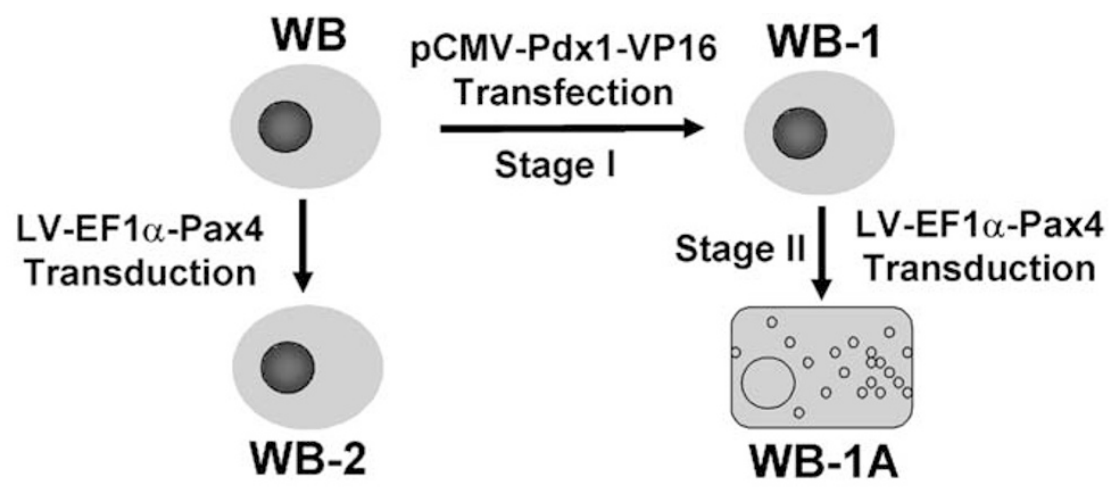

b
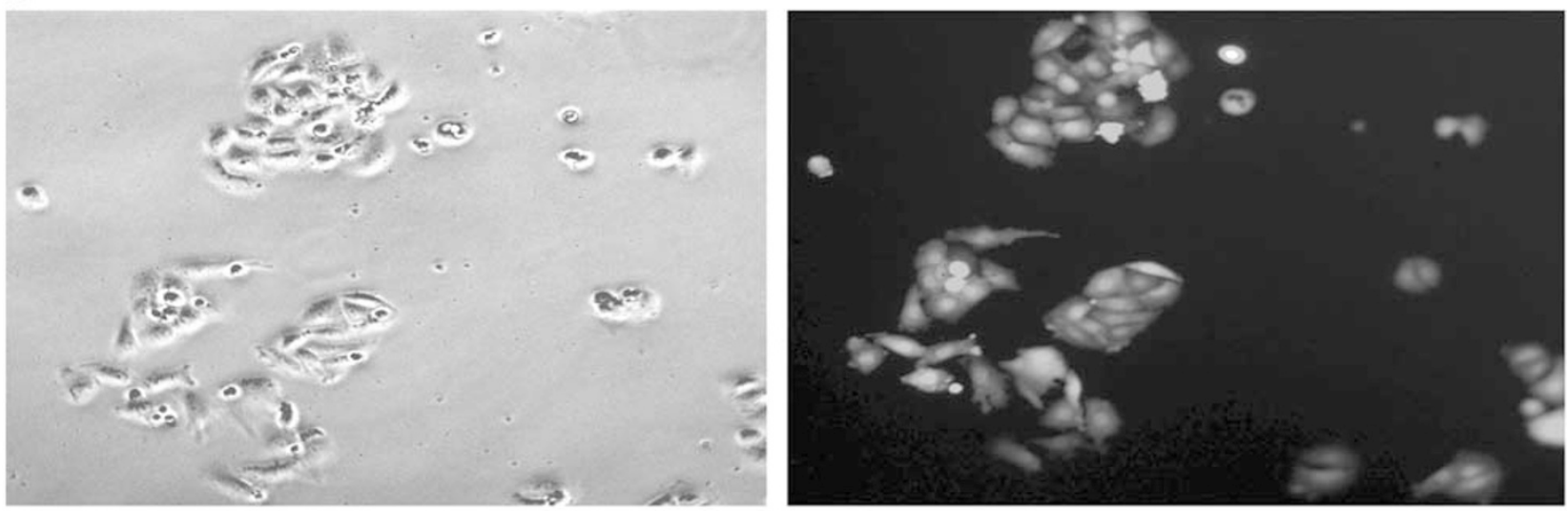

Figure 1 (a) Strategy of generating specified cell lines. WB-1 cells containing both Pdx1-VP16 and RIP-GFP were previously described. ${ }^{9}$ WB-1A and WB-2 cells were obtained by transducing the WB-1 and WB cells with LV EF1 $\alpha$-Pax4, respectively. WB cells transduced with LV-EF1 $\alpha$-GFP served as lentiviral vector control for this study. (b). Transduction of WB cells with LV-EF1 $\alpha$-GFP. To evaluate the transduction efficiency, WB cells were transduced with LV-EF1 $\alpha$-GFP at a MOI of 20 for $48 \mathrm{~h}$ and the transduction efficiency is nearly $100 \%$ (GFP-positive cells by fluorescence). Lens $(\times 40)$ was used in original magnification.

a

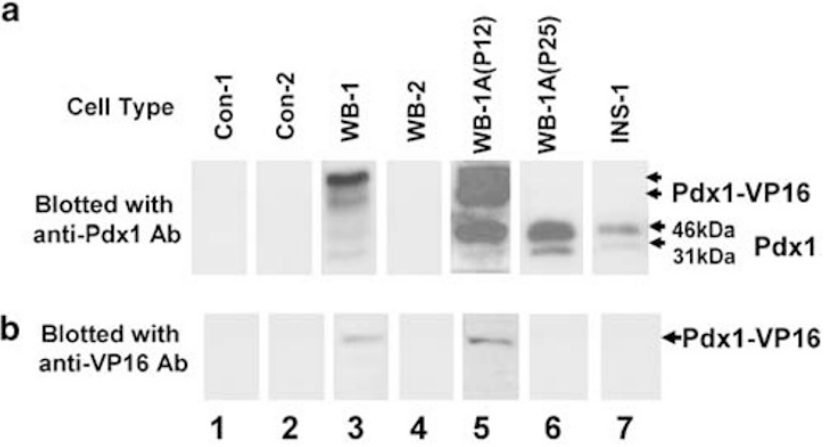

Figure 2 Expression of Pdx1 and Pdx1-VP16 proteins. Cell lysates (50 $\mu \mathrm{g} / \mathrm{lane})$ extracted from WB-1, WB-1A, WB-2, WB, and $10 \mu \mathrm{g}$ cell lysates from INS-1 cells were separated by SDS-PAGE. Pdx1 and Pdx1-VP16 proteins were detected by Western blotting with antiPdx1 (a) antibody (1:5000, CV Wright), and anti-VP16 (b) antibody (1:200, BD), respectively. Arrows indicated two forms of Pdx1 proteins and Pdx1-VP16 fusion proteins (phosphorylated upper and dephosphorylated lower bands, respectively). The antibodies used for each Western blot were indicated on the left-side of each panel. Con-1, WB cell transfected by empty plasmid vector; Con-1, WB cells transduced with lentiviral vector-GFP; WB-1A (P12)-WB-1A cells at passage 12; and WB-1A (P25)-WB-1A cells at passage 25.

WB-1 (Passage 11) and WB-1A (passage 12) cells expressed the fusion protein of Pdx1-VP16 (arrows, lanes 3 and 5, 2a and 2b) as well as weak bands of endogenous Pdx1 in WB-1 cells (Figure 2a, Lane 3). Interestingly, activation of Pax4 in WB-1 cells resulted in high-level expression of endogenous Pdx1 proteins (Figure 2a, lane 5, strong $46 \mathrm{kDa}$ band and weak $31 \mathrm{kDa}$ band) and complete loss of exogenous Pdx1-VP16 expression at passage 25 of WB-1A cells (Figure 2a and b, lane 6). This finding is consistent with the report that $\mathrm{Pdx} 1$ protein has two forms: a predominantly inactive $31-\mathrm{kDa}$ form in the cytoplasm as well as active $46-\mathrm{kDa}$ form in the nucleus. ${ }^{23}$ High-glucose treatment of cells also stimulates translocation of $\mathrm{Pdx} 1$ from the cytoplasm to the nucleus of human pancreas $\beta$-cells. ${ }^{23}$ In addition, this observed pattern of two Pdx1 forms is consistent with the pattern of a mature $\beta$-cell $\mathrm{Pdx} 1$ expression observed in INS-1 cells (lane 7, only loading $10 \mu \mathrm{g}$ cell lysates). No Pdx1 or Pdx1-VP16 bands were detected in WB-2 cells (lane 4), empty plasmidtransfected (lane 1), or LV-GFP-transduced (lane 2) control WB cells. These results indicated that activation of Pax4 in WB-1A cells upregulated the expression of endogenous activated $46 \mathrm{kDa}$ form of Pdx1.

\section{Comparison of Gene Expression Profiles}

We previously demonstrated by the gene expression profiling of newly generated WB-1 cells that they 
were unable to activate the late-stage pancreatic transcription factors Pax4, Pax6, MafA, and Isl-1, nor the $\beta$-cell function-related genes SUR1, Kir6.2, SNAP25, and IAPP, without further differentiation. ${ }^{9}$ Pax4 expression has been proposed to occur after $N K \times 2.2$ expression during the development of pancreatic $\beta$-cells. ${ }^{10}$ Hence, to determine the role of Pax4 in the liver-to-endocrine pancreas transdifferentiation as well as to explore the underlying molecular mechanisms of this process, we compared the gene expression profiles among WB-1, WB-1A, WB-2, WB-GFP, and INS-1 cells by RT-PCR. As shown in Figure 3, following Pax4 activation (down arrows) in WB-1A cells, genes (Pax6, Isl-1, and $M a f A$ ) related to late-stage $\beta$-cell development (Figure 3a) and genes (SUR1, SNAP25, Kir6.2, and $I A P P)$ related to $\beta$-cell function and insulin secretion (Figure $3 \mathrm{~b}$ ) were activated (up-arrowheads). The gene expression profile for WB-1A cells resembles that of functional $\beta$-cell insulinoma INS-1 cells and post-transplanted WB-1 cells, as demonstrated previously, ${ }^{9}$ suggesting that $\mathrm{WB}-1 \mathrm{~A}$ cells have a mature $\beta$-cell gene expression profile. Moreover, Pax4 overexpression in WB-1A cells significantly downregulated $N k x 2.2$ expression (indicated by star), possibly by a negative feedback mechanism, since $N k x 2.2$ is positioned immediately upstream of Pax4 in $\beta$-cell development. ${ }^{10}$ In contrast, Pax4 alone in WB-2 cells only activated Isl-1, MafA, and glucagon, suggesting that these genes may be the targets of Pax4 (see Discussion), while activation of Pax6, SUR1, Kir6.2, SNAP25, or IAPP appears to require active participation of other pancreatic transcription factors. We also examined these cell lines with respect to the expression of liver genes for albumin, CK19, TGF- $\alpha$, and hexokinase, using rat liver RNAs as positive controls. Figure 3c shows that albumin and the liver stem-cell marker CK19 were expressed in parental WB cells, but were undetectable in WB-1, WB-1A, and WB-2 cells. Interestingly, TGF- $\alpha$ was weakly expressed in WB and WB-1 cells, but was upregulated in WB-1A and WB-2 cells, suggesting that overexpression of Pax4 in WB-1A and WB-2 cells may be responsible for the TGF- $\alpha$ upregulation. No change for hexokinase expression is observed in all WB-derived cells.

\section{Pax4 Protein Binding to Rat Insulin I Gene}

While we observed strong Pax4 gene expression in Figure 3a (down arrows) after transduction of LVPax4 into WB or WB-1 cells, it was essential to learn whether the resulting WB-2 and WB-1A cells expressed functional Pax4 protein and whether the expressed protein could bind to its target DNA. To address these questions, we biotin-labeled $24 \mathrm{bp}$ oligonucleotides of rInsIC2 element containing the potential high-affinity Pax4 binding sequences as an EMSA probe. Nuclear extract was obtained from WB-derived cells to determine the Pax4-DNA bind- ing characteristics. Purified Pax4 protein was used as positive control to indicate the location of the Pax4:rInsIC2 complex. As shown in Figure 4a, the rInsIC2 probe was found to bind to both purified Pax4-V5 fusion protein and exogenous mouse Pax4 protein in WB-2 and WB-1A cells with comparable affinity. The rInsIC2 sequence exhibits very high affinity for Pax4 protein, ${ }^{14}$ and unlabeled rInsIC2 duplex was found to be a highly effective competitor, as evidenced by its ability to abolish Pax4 binding to the biotin-labeled probe (see Figure 4a, lane 3 in WB-2 and WB-1A cells, as well as purified Pax4 protein used as a positive control). To exclude the possibility of the nonspecific, dilution effect due to an excess amount (200-fold) of unlabeled probe, we used biotin-labeled EBNA probe (provided by manufacturer in the kit) and biotin-labeled rInsIC2 probe to test their specific binding ability to their nuclear target proteins in the presence of specific and nonspecific unlabeled binding probes. Figure $4 \mathrm{~b}$ shows that the nonspecific excessive unlabeled probes failed to displace the specific binding of EBNA or rInsIC2 to their own target nuclear proteins (lane 4). These results confirmed that LV-mediated Pax4 protein in WB-2 and WB-1A cells specifically binds to the rInsIC2 element with high affinity, indicating that exogenous Pax4 mediated by lentivector is indeed biologically functional.

\section{Insulin Protein Expression}

Given the distinct patterns of Pdx1 protein expression as well as gene expression pattern differences between WB-1 and WB-1A cells, we attempted to compare the levels of expression of insulin and glucagon by immunocytochemistry (Figure 5). Cells were double-stained with anti-insulin and antiglucagon antibodies and corresponding fluorescent-dyelabeled secondary antibodies. As expected, WB-1A cells (at passage number 11) showed intense cytoplasmic insulin staining (red) as did in INS-1 cells, while WB-1 cells (at passage number 10) without further differentiation exhibited very weak insulin staining. No insulin was detected in WB-2 and WBGFP (lentivector control) cells. In contrast, glucagonstaining cells were not seen in any of the examined cells. Rat pancreatic islets (Figure 5, upper left) served as positive controls for insulin-positive $\beta$-cells (red) and glucagon-positive $\alpha$-cells (green).

\section{Functional Characterization In Vitro}

To learn whether WB-1A cells were functional with respect to insulin release, we examined in vitro insulin release upon glucose challenge. The cells were stimulated with $20 \mathrm{mM}$ D-glucose medium for $2 \mathrm{~h}$ after overnight incubation of the cells with $0.5 \%$ FCS in a low glucose $(3.0 \mathrm{mM})$ medium. The culture media and cell lysates were collected and subjected to ELISA for insulin. Table 1 summarizes the insulin content and release in WB, WB-1, WB-1A, and WB-2 


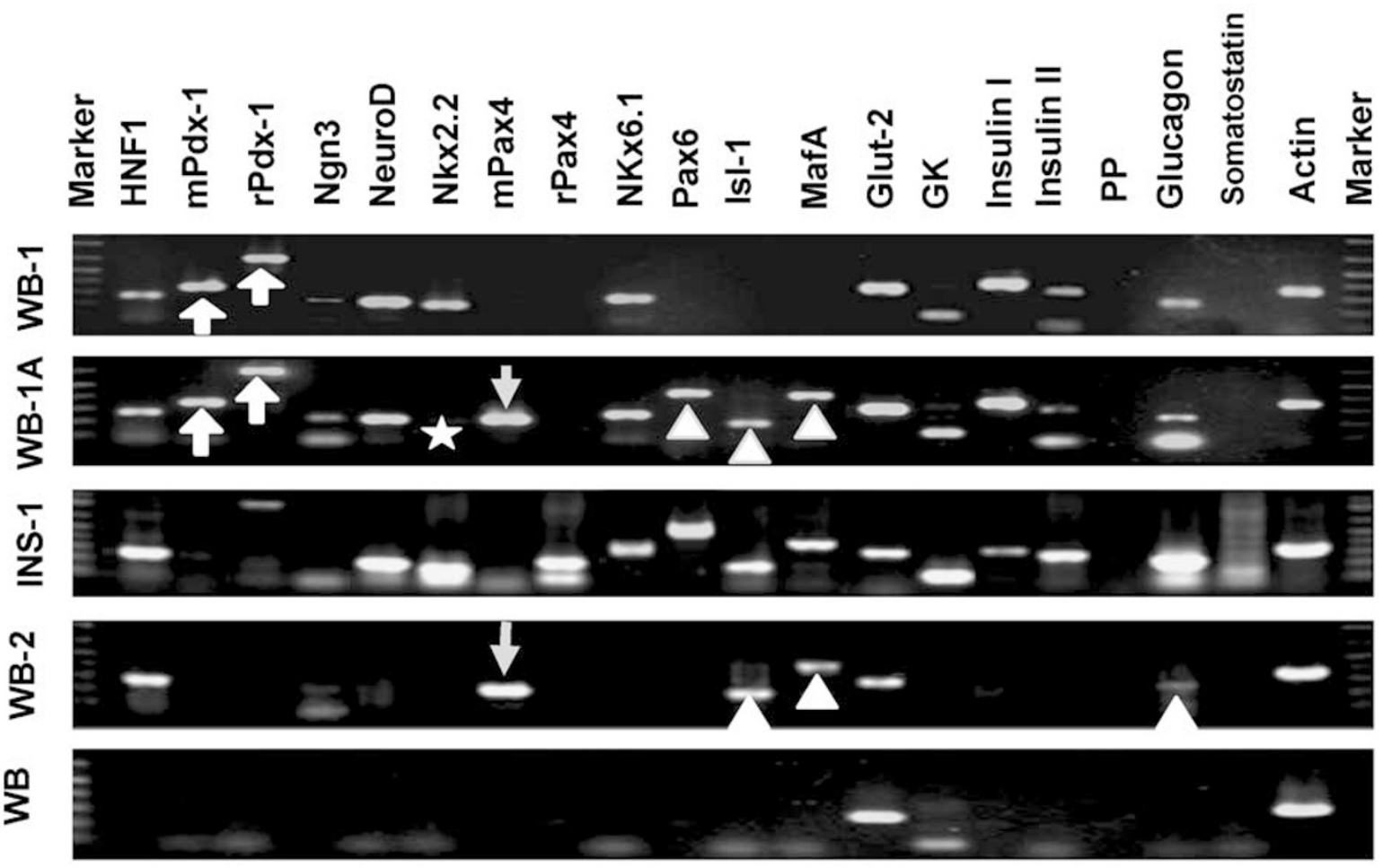

b

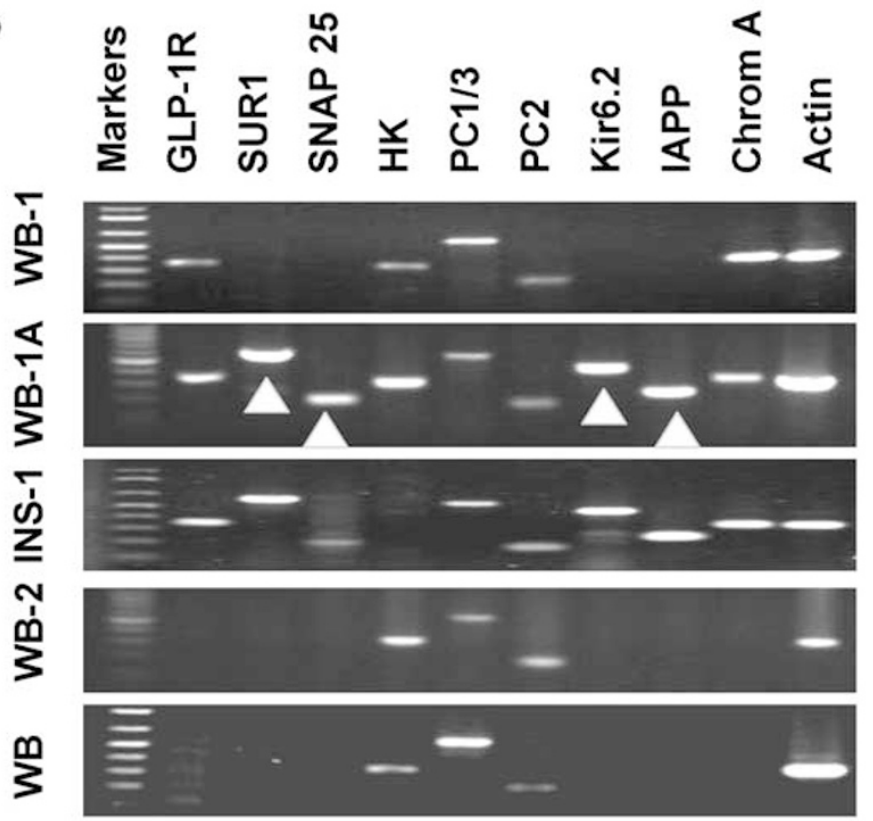

C

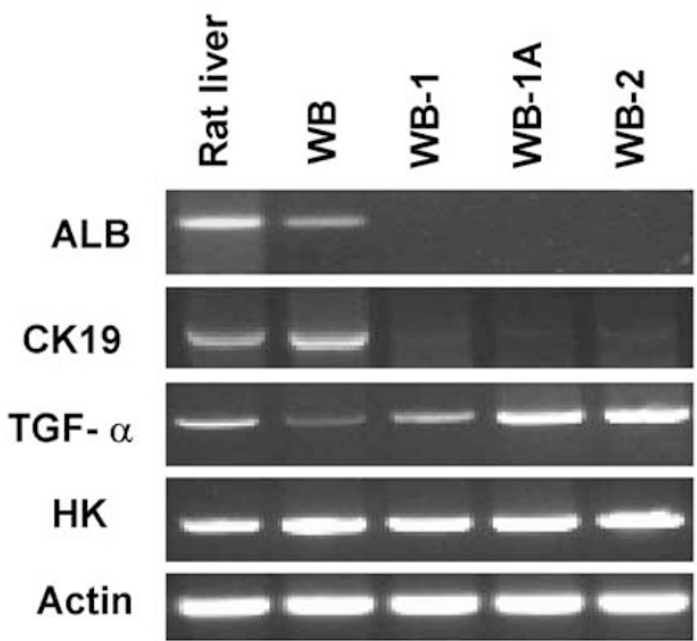

Figure 3 Comparison of $\beta$-cell related gene expression profiles among the WB-derived cell lines (a and b). Total RNA was extracted from the cells and RT-PCR was performed. All primers (Table 1, Supplementary data) were designed across intron(s). INS-1 (clone 823/13) cells were used as $\beta$-cell positive control. Up arrows indicate exogenous ( $m P d x 1)$ and endogenous $(r P d x 1)$ expression in WB-1 and WB1A cells. Down arrows indicate exogenous Pax4 expression. Arrowheads indicate newly activated genes. Star indicates the down regulation of $N k x 2.2$ in WB-1A cells. Note that certain lanes contain two bands and the lower bands represent the primer dimers. (c) Expression of liver-related genes in WB-derived cells. Expression of the liver-related genes including albumin (ALB), cytokeratin 19 (CK19), transforming growth factor- $\alpha$ (TGF- $\alpha)$ and hexokinase (HK), among WB and WB-derived cells was determined by RT-PCR. As noted in (c), $A L B$ and $C K 19$ were expressed in parental WB cells, but they were not detectable in the WB-1, WB-2, and WB-1A cells. There is upregulation of $T G F-\alpha$ expression in WB-2 and WB-1A cells. No change is noted in hexokinase gene expression.

cells upon glucose stimulation. These results indicate that the WB-1A cells indeed are able to respond to a glucose challenge, as evidenced by stimulated insulin release, although the amount of released insulin is much less (approximately 1\%) than that of INS- 1 cells $(405 \pm 35 \mathrm{ng} / \mathrm{mg}$ protein/ $2 \mathrm{~h})$. The $2 \times$ 
a

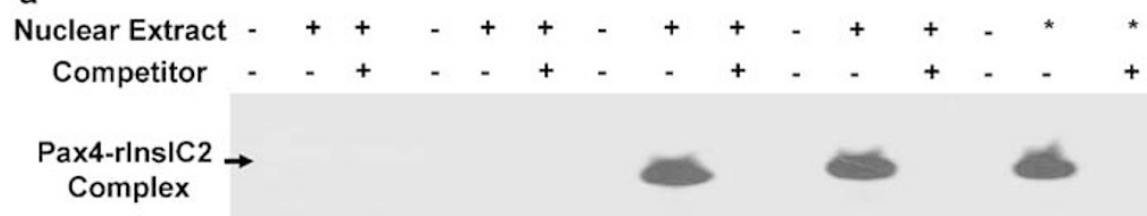

Labeled

Free Probe

(rInsIC2)

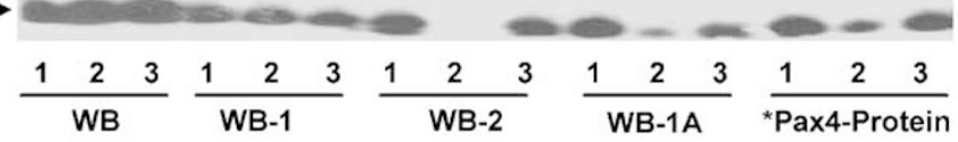

b

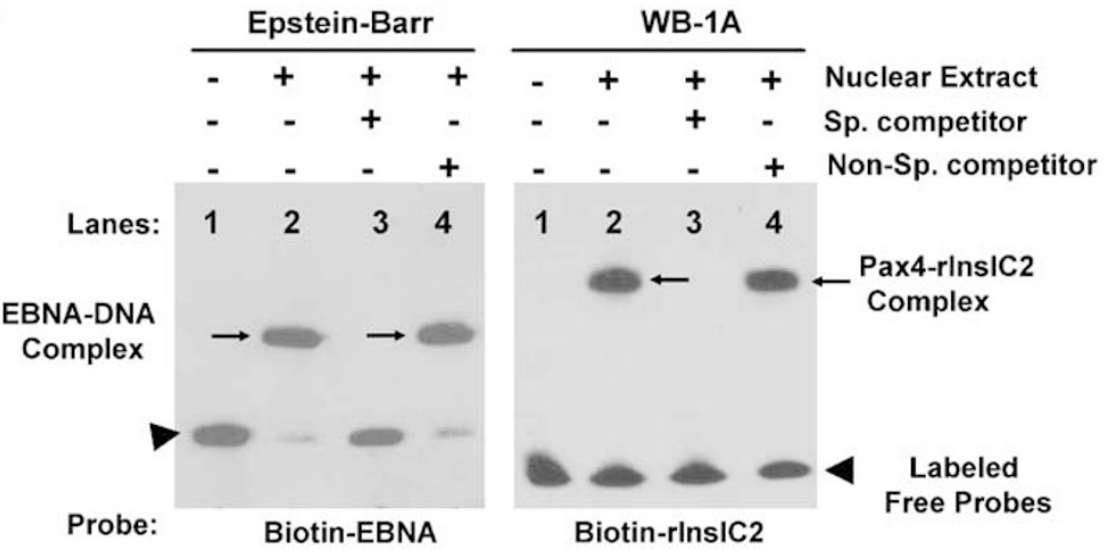

Figure 4 Chemiluminescent EMSA of rInsIC2-DNA:Pax4-protein complexes. (a) The probe used here was 24-bp biotin-labeled rat insulin I C2 element (rInsIC2) DNA duplex, and nuclear extracts from WB, WB-2, WB1A, and WB-1 cell were the source of the Pax4 transcription factor. Purified Pax4 protein $\left({ }^{*}\right)$ serves as a positive control to indicate the Pax4 position in EMSA. When used, unlabeled specific competitor rInsIC2 duplex was present at a 200-fold molar excess over labeled rInsIC2 target to eliminate detected specific shift. X-ray film was exposed for 1 min. The upper arrow indicates the complex formed by Pax4 protein and the rInsIC2 duplex, and the arrowhead indicates free biotin-labeled rInsIC2 DNA probe. For each trio: Lane 1, free probe; Lane 2, probe plus nuclear extract; and Lane 3 , probe plus nuclear extract in the presence of excess unlabeled rInsIC2 competitor. (b) Exclusion of nonspecific dilution effect by excessive unlabeled probes. Two biotin-labeled DNA probes (Epstein-Barr nuclear antigen- EBNA (left), and rInsIC2 (right)) were used to test the binding specificity to their specific target proteins in the nuclear extracts in the presence of specific or nonspecific excessive (200-fold) unlabelled probes as indicated in the figure. Unlabelled DNA probe for EBNA binding serves as specific probe and unlabelled rInsIC2 DNA probe uses as nonspecific competitor for biotin-EBNA probe, respectively, and vice versa. Sp, specific; Non-Sp, nonspecific.

increase in insulin release into the medium after glucose stimulation of WB-1A cells is statistically significant $(P<0.001)$. However, newly generated WB-1 cells (passage 10) were incapable of releasing insulin upon glucose stimulation and contained very low insulin levels. No insulin synthesis or release was detected in WB and WB-2 cells.

\section{In Vivo Cell Transplantation}

We previously demonstrated that implanted WB-1 cells can reverse hyperglycemia in diabetic animals, ${ }^{9}$ only after 2-3 weeks to allow for WB-1 cells to undergo further differentiation and maturation in vivo into fully functional $\beta$-like cells. To further examine differences in the way those cells reverse hyperglycemia, WB-1, WB-1A, WB-2, and WB cells were transplanted into the left renal capsular spaces of Stz-induced diabetic NOD-scid mice. Left nephrectomy was performed around day -40 after transplantation in mice receiving WB-1 and WB-1A cells, and the explanted tissues were analyzed by morphologic and immunologic evaluation. Figure 6 shows that transplantation (Tx) of either WB-1 or WB-1A cells reduced blood glucose levels in diabetic mice. However, mice implanted with WB$1 \mathrm{~A}$ cells required approximately 10 days to reduce the blood glucose level from $\sim 400$ to $\sim 200 \mathrm{mg} / \mathrm{dl}$, whereas 20 days were necessary for mice transplanted with WB-1 cells to reach similar levels. Furthermore, after 21 days in vivo, WB-1A cells completely normalized blood glucose levels and maintained this euglycemia up to the time of nephrectomy (Figure 6a, square line). The WB-1 cells reduced blood glucose levels to $200 \mathrm{mg} / \mathrm{dl}$, but did not normalize blood glucose level at day 40 posttransplantation (Figure 6b triangle line). Statistical 

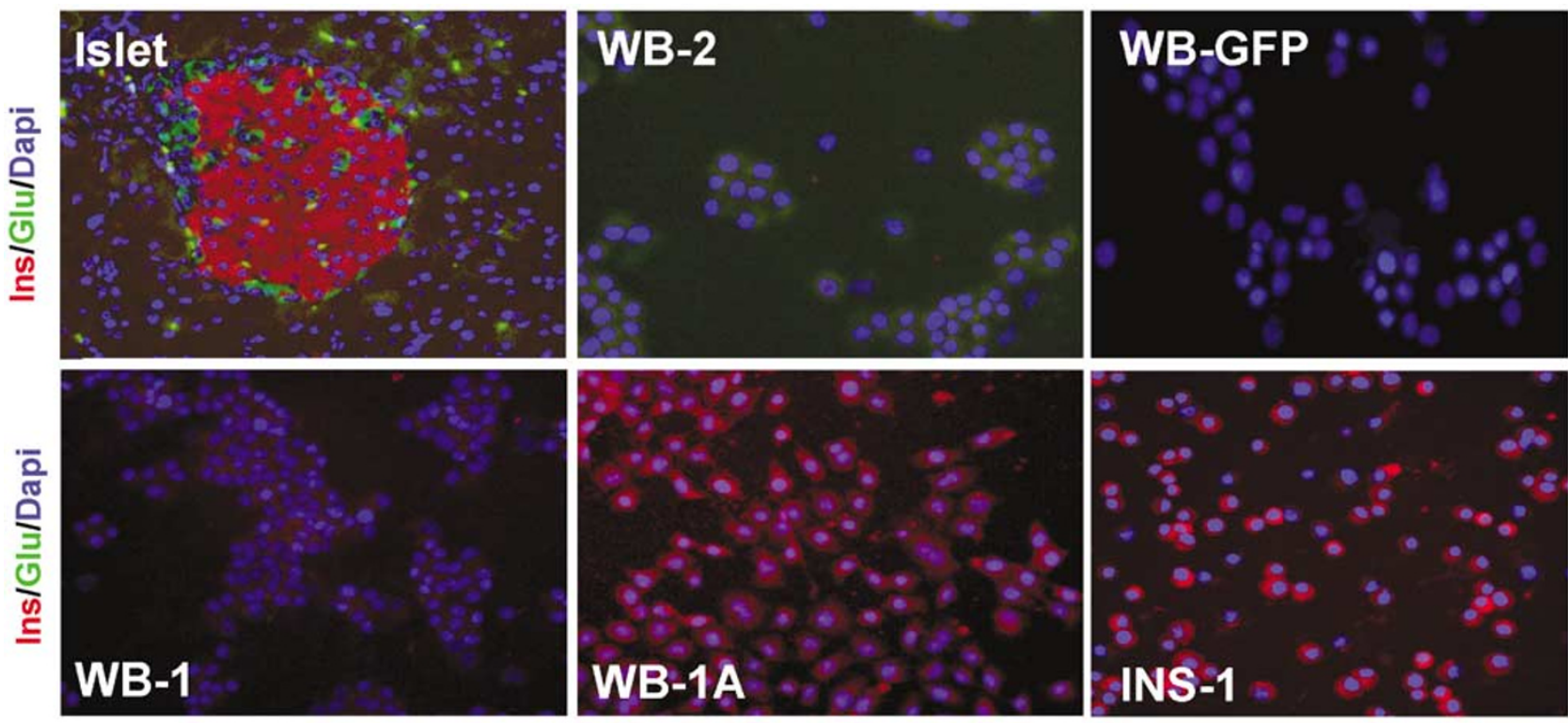

Figure 5 Detection of insulin and glucagon by immunocytochemistry. Slides made from newly transfected WB-1 cells, transduced WB1A, WB-2, and WB-GFP cells were stained with anti-insulin (1:500) and antiglucagon (1:100) antibodies. Insulin staining was shown in red, glucagon in green, and nuclei in blue (Dapi). Rat pancreatic islet and INS-1 cells served as positive controls for insulin and glucagon (rat islet). $\times 40$ original magnification.

Table 1 Insulin content and insulin release during $2 \mathrm{~h}$ after glucose stimulation

\begin{tabular}{lcccc}
\hline Cell & $\begin{array}{c}\text { Insulin content } \\
(\mathrm{ng} / \mathrm{mg} \text { protein) }\end{array}$ & \multicolumn{2}{c}{ Insulin release $(\mathrm{ng} / \mathrm{mg}$ protein/2 $\mathrm{h})$} & $\begin{array}{c}\text { Fold of insulin release 20 mM } \\
\text { Glucose }(+) /(-)\end{array}$ \\
\cline { 3 - 4 } & & $20 \mathrm{mM}$ glucose (-) & $20 \mathrm{mM}$ glucose (+) & 0.98 \\
WB & $0.26 \pm 0.01^{*}$ & $0.40 \pm 0.01^{*}$ & $0.39 \pm 0.01^{*}$ & 1.06 \\
WB-1 & $2.73 \pm 0.1$ & $0.79 \pm 0.02$ & $0.84 \pm 0.02$ & 1.00 \\
WB-2 & $0.50 \pm 0.02^{*}$ & $0.47 \pm 0.01^{*}$ & $0.47 \pm 0.02^{*}$ & 2.04 \\
WB-1A & $22.0 \pm 0.13$ & $1.66 \pm 0.01$ & $3.38 \pm 0.05^{* * *}$ & \\
\hline
\end{tabular}

${ }^{*}$ No statistical significance from blank or cell culture medium readings, ${ }^{* *} P<0.001$

WB-1 cells represent newly generated Pdx1-VP16 expressing cells at passage 10-11 with no in vitro further differentiation.

analysis at day 21 post-transplantation reveals that there is a statistical significance $(P<0.05)$ in the blood glucose levels between WB-1 and WB-1A cell-transplanted mice. IPGTT was performed in WB-1, WB-1A transplanted mice after their blood glucose levels were normalized and one day before nephrectomy was performed, and they showed a glucose-responsive time course similar to that of normal mice (Figure 6b). Removal or explantation (Ex) of implanted WB-1 and WB-1A cells by left nephrectomy approximately day -40 post-transplantation induced a persistent return to hyperglycemia (Figure 6a, down arrows), confirming that the implanted cells were indeed responsible for the reduction of blood glucose levels. As expected, WB and WB-2 transplanted mice remained hyperglycemic and were killed approximately day -40 post-transplantation. The pancreata in all planned killed mice were examined for $\beta$-cell regeneration by an immunostaining with anti-insulin antibody, and the results indicated only scattered insulin-positive cells present in some mice, indicating no significant $\beta$-cell regeneration in all mice in this study. In animals not undergoing scheduled killing, after 2 months, mice receiving WB-1 cells gradually normalized blood glucose levels and these mice remained euglycemic ( $\sim 100 \mathrm{mg} / \mathrm{dl})$ up to 4 months post-transplantation, as previously described. ${ }^{9}$ To our surprise, the four remaining mice transplanted with WB-1A cells became hypoglycemic ( $\sim 40 \mathrm{mg} / \mathrm{dl})$ after 2 months and died soon thereafter. These results indicate that both WB-1 and WB-1A cells can reverse hyperglycemia, WB-1 cells behave as $\beta$-cell precursors as in vitro, whereas WB-1A cells behave as mature, but dysregulated $\beta$-like cells.

\section{Histology and Insulin Production in Explanted Cells}

To examine morphologic characteristics and insulin production in the explanted cells at the time of nephrectomy, we examined the cytological features of WB-1A cells by H\&E staining (Figure 7a, upper 

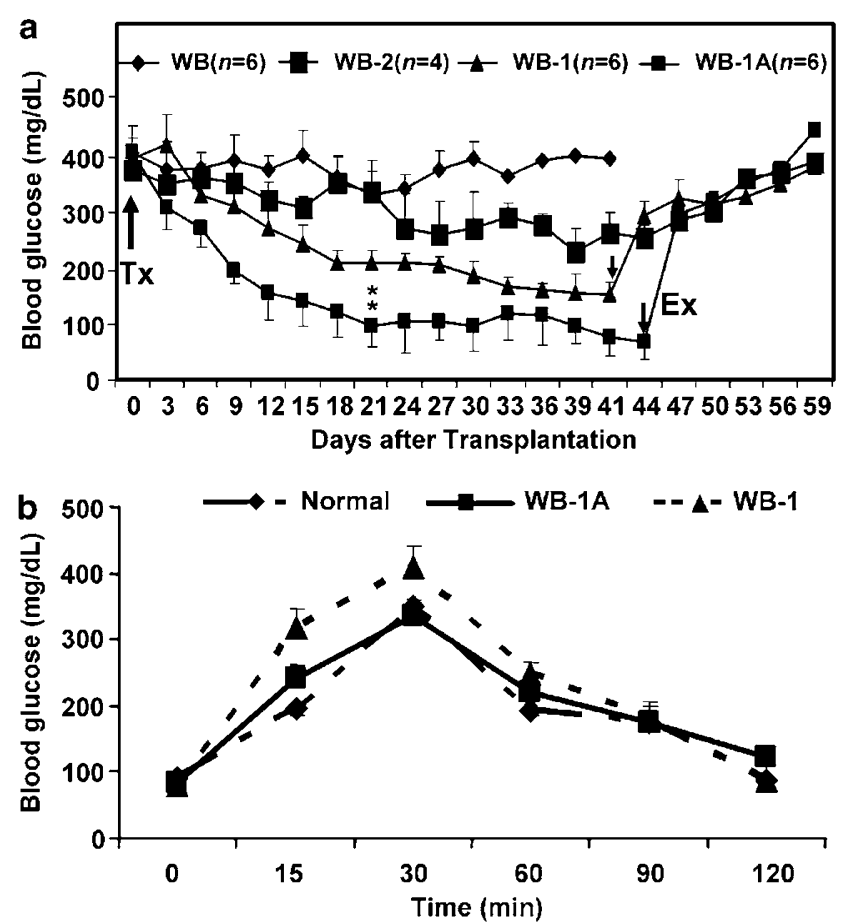

Figure 6 (a) In vivo cell transplantation. WB, WB-1, WB-1A or WB-2 cells $\left(1 \times 10^{6} /\right.$ mouse $)$ were implanted under the left subrenal capsules of Stz-treated diabetic NOD-scid mice (up arrow, $\mathrm{Tx}=$ transplantation). The blood glucose levels were monitored under nonfasting conditions. The left kidney from the transplanted mice was removed around day-40 post-Tx (down arrow, Ex $=$ explanation). ${ }^{*} P<0.05$. (b). Results of IPGTT in normoglycemic WB-1A transplanted $(n=3) x$ days after transplantation, WB-1 $(n=3)$ y days after transplantation, as well as normal $(n=3)$ NOD-scid mice.

panel) and the amount of insulin by immunostaining (Figure 7a, lower panel). The explanted WB-1A cells exhibited neuroendocrine cytology similar to pancreatic islet cells, and contained abundant insulin in nearly $100 \%$ of the cells, with the intensity of insulin staining equivalent to islet $\beta$ cells. Additionally, comparison of the intensity of insulin immunostaining between WB-1 (Figure 7b4) and WB-1A (Figure 7b-5) cells approximately day -40 post-transplantation demonstrated that WB-1A cells produced much more insulin, which is consistent with their effectiveness in reducing hyperglycemia in diabetic mice. No insulin production was detected in either WB (Figure 7b-1) or WB-2 cells (Figure 7b-2). No amylase was detected in WB1A cells (Figure 7b-7) with mouse pancreatic exocrine tissue as positive control (Figure 7b-6). These results demonstrate WB-1A cells have the characteristic morphology of mature $\beta$-like cells and produce large amounts of insulin, as compared to WB-1 cells at the same time post-transplantation.

\section{Discussion}

The primary goal of this study was to determine whether pancreatic transcription factor Pax4, acting alone or in the presence of Pdx1-VP16, could convert rat hepatic stem-like WB cells into functional insulin-producing cells in vitro. Activation of Pax4 in WB-1A cells resulted in the activation of multiple downstream late-stage transcription factors and other genes related to $\beta$-cell function and insulin secretion, whereas these genes were silent in the $P d x 1-V P 16$ expressing pancreatic precursorlike WB-1 cells prior to in vitro or in vivo further differentiation. ${ }^{9}$ Dissection of the molecular events occurring in WB-1A cells showed that expression of Pax4 led to the activation of multiple key genes (ie, Pax-6, MafA, Isl-1, SUR1, Kir6.2, SNAP25, and $I A P P)$, setting the stage for fully differentiated, functional pancreatic $\beta$-like cells to emerge in vitro. The WB-1A cells produced and released insulin upon in vitro glucose stimulation, without the need for further in vitro differentiation or in vivo cell transplantation. These cells also exhibited a gene expression profile nearly identical to post-transplanted WB-1 cells, ${ }^{9}$ and very similar to that of functional rat INS-1 insulinoma cells. WB-1A cells also produced similar intensity of insulin compared to native mouse islet beta cells following transplantation into diabetic mice and reversed hyperglycemia in these animals without the typical delay required for in vivo WB-1 cell differentiation. ${ }^{9}$ Therefore, we conclude that expression of Pax4 in Pdx1-VP16expressing hepatic WB cells is able to activate late-stage genes and generate glucose-responsive insulin-producing cells.

Little is known regarding the transcription factor cascade engaged during the conversion of hepatic cells into functional $\beta$-cell-like insulin-producing cells. We previously showed ${ }^{9}$ that introduction of Pdx1-VP16 into hepatic WB cells initiates earlystage $\beta$-cell differentiation, resulting in the activation of HNF1, endogenous Pdx1, Ngn3, NeuroD/ Beta2, $N K \times 2.2$, and $N K \times 6.1$ as well as genes related to pancreatic endocrine function (eg, insulin $I \& I I$, glucagon, Glut-2, GK, GLP-R, PC1/3, PC2, and ChromA). However, Pdx1-VP16 alone could not activate such late-stage pancreatic transcription factors as Pax-4, Pax-6, MafA, and Isl-1, as well as the $\beta$-cell function-related genes SUR1, Kir6.2, SNAP25, and IAPP in newly generated WB-1 cells. The aforementioned genes become activated only after further differentiation in vivo, when WB-1 cells were transplanted into Stz-induced diabetic NODscid mice. ${ }^{9}$ The WB-1 cells can also become functional upon long-term in vitro culture in highglucose medium. ${ }^{9}$ The shift of WB-1 cells from glucose-insensitive to glucose-responsive phase appears to be triggered by hyperglycemia or highglucose culture conditions.

Pax4 is positioned in the upper hierarchy among the $\beta$-cell transcription factors that are silent in WB-1 cells. Its expression in $\beta$-cell precursors results in the differentiation and maturation of $\beta$ like cells. ${ }^{10}$ Recently, the concerted action of Pax4 and Nkx2.2 was shown to be essential for onset of 


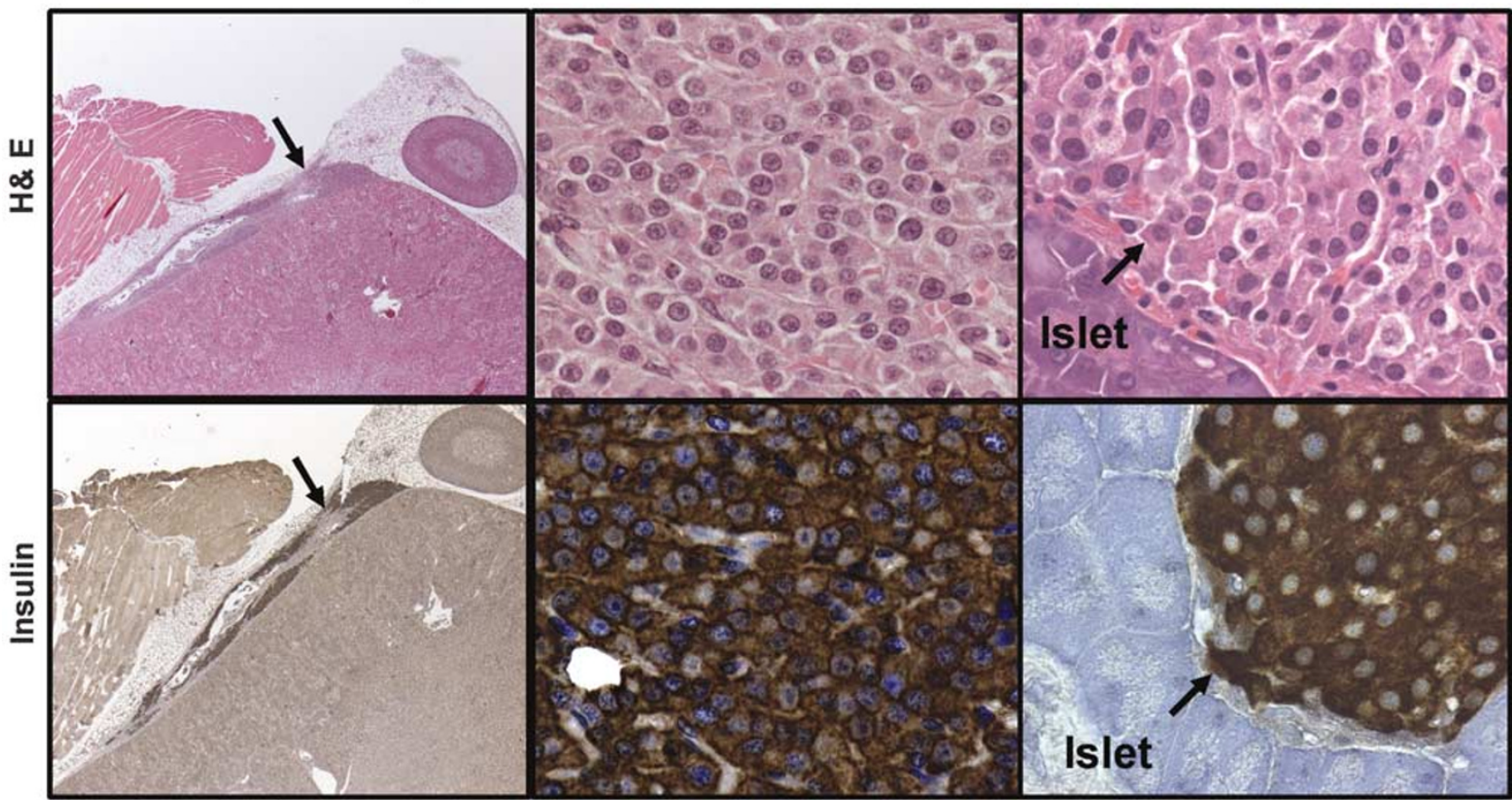

b Explanted WB-derived cell lines

Pos. Control

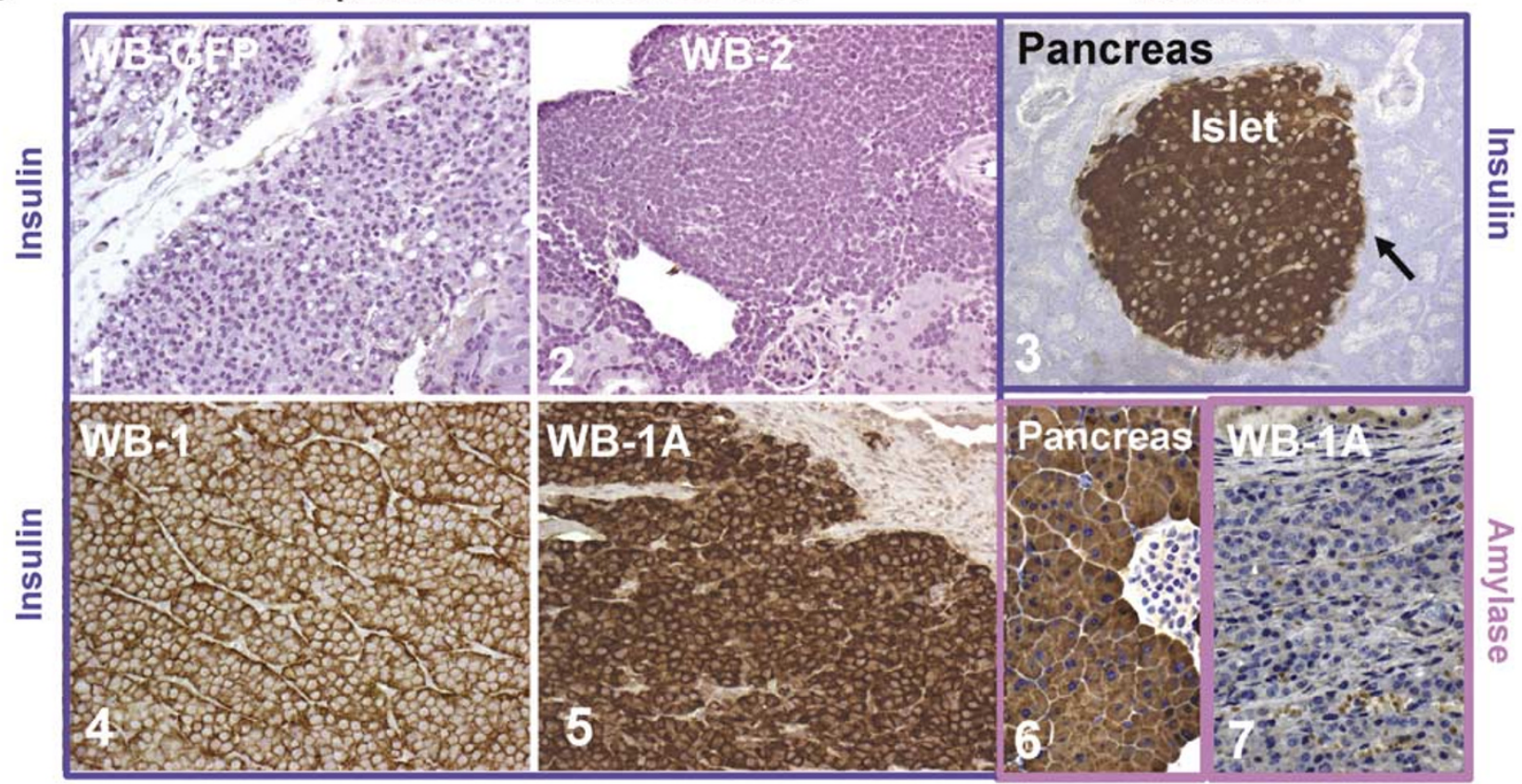

Figure 7 Histology and insulin production evaluation. (a) Histology, cytology, and insulin production in the explanted WB-1A cells. H\&E (upper) and insulin staining (1:500) (lower) were performed on paraffin sections of the explanted WB-1A cells (day -42 post-Tx). Arrows indicate the renal subcapsular space filled with transplanted WB-1A cells. Pancreas served as controls. (b). Immunohistochemistry of insulin and amylase. Diabetic NOD-scid mice received renal subcapsular transplants of WB-derived (WB, WB-2, WB-1, or WB1A) cells. Upon explantation, these explanted tissues were stained with anti-insulin antibodies (1:500; panels 1-5). Pancreatic islets served as insulin positive control (panel 3). WB-1A explanted tissue was also stained with antiamylase (panel 7) antibody (dilution 1:300) and showed no detectable amylase in WB-1A cells. Pancreas serves as positive control for amylase (panel 6). Original objective lens are $\times 2.5$ (Figure 1a, left panel), $\times 100$ (Figure 1a, middle and right panels), and $\times 40$ (Figure 1b)

pancreatic $\beta$-cell differentiation. ${ }^{24}$ By inactivating Pax4 in newborn Pax4-deficient mice, pancreata are almost entirely devoid of mature $\beta$-cells, suggesting that Pax4 is crucial for the formation of mature insulin-producing $\beta$-cells. ${ }^{11}$ However, loss of functional Pax4 does not affect the expression of Ngn3, 
Islet1, Nkx2.2 or Pax6 in pancreatic precursors, ${ }^{24}$ suggesting that Pax4 is involving in late-stage of $\beta$-cell differentiation and maturation. Indeed, as demonstrated in the present report, forced Pax4 expression in WB-1 cells activated Pax6, Islet1, and MafA, and promoted the cells to undergo further differentiation and maturation. However, overexpression of Pax4 alone in WB-2 cells only activated Isl-1, MafA, and glucagon genes without activating Pax6 and other function-related genes SUR1, Kir6.2, SNAP25, and IAPP, suggesting that Isl-1, MafA, and glucagon genes may be direct targets for Pax 4 and that the expression of Pax6, SUR1, Kir6.2, SNAP25, and IAPP genes requires participation of other gene and proper external factors in addition to Pax4.

It has been reported that Pax4 overexpression in mouse embryonic stem cells increases insulinproducing cells dramatically, as compared to Pdx1expressing mouse embryonic cells. ${ }^{25}$ However, our results indicated that an appropriate background of activated genes related to $\beta$-cell development is required for Pax4 to promote $\beta$-cell maturation in the context of the liver-to-endocrine pancreas transdifferentiation. It is unclear whether ectopic expression of Pax4 in WB liver-stem cells reproducibly results in weak expression of glucagon. One possibility is that the expression of glucagon is related to $\beta$-cell development in the developing endocrine pancreas. It has been well documented by others that glucagon and insulin genes are coexpressed in early pancreatic endocrine precursor cells prior to terminal differentiation. Glucagon and insulin gene expression is both selectively increased and extinguished as development proceeds, concomitant with a restriction to the patterns of expression characteristic of mature islet cell types. ${ }^{26}$ We have previously shown that short-term (6 days) expression of either Pdx1 or Pdx1VP16 activated both insulin II and glucagon genes in WB cells. However, exclusive expression of insulin and loss of glucagon expression occurred only after long-term expression of $\mathrm{Pdx} 1$ or Pdx1VP16 in these cells. ${ }^{21}$

Based on our data, the molecular events for Pdx1VP16-induced transdifferentiation of hepatic stemlike WB cell into functional insulin-producing cells can be summarized as follows. Expression of $P d x 1$ VP16 converts the hepatic stem-like WB cells into pancreatic precursor cells characterized by stagespecific transcription factor expression. However, these precursor cells cannot respond to glucose challenge in vitro without further differentiation. Generation of glucose-responsive liver-derived insulin-producing cells from these precursor cells appears to require: (a) a long-term culture in a high-glucose medium; (b) transplantation of the cells into diabetic mice; or (c) introduction of Pax4 into the $P d \times 1-V P 16$-expressing cells. While questions remain concerning the role of Pax4 in the liver-to-endocrine pancreas transdifferentiation, it appears in our system that Pax4 expression precedes and facilitates Pax6 expression in the Pdx1-VP16mediated pancreatic transdifferentiation and that consequential effect of constitutive Pax 4 activation appears to result in down-regulation of $N k \times 2.2$ gene expression.

The results of previous studies suggest that Nkx6.1 was likely to be an immediate downstream target gene of Pax4 during normal embryogenesis. ${ }^{10}$ We find, however, that $N k x 6.1$ can be activated in the absence of Pax4 expression, suggesting that Nkx6.1 might act upstream of Pax4 in the cascade of transcription factor activation in the liver-to-endocrine pancreas transdifferentiation. Interestingly, a similar gene expression profile (showing Pax4 activation, along with other transcription factors Pax6, Isl-1, and MafA as well as $\beta$-cell functionrelated genes SUR1, Kir6.2, SNAP25, and IAPP in WB-1 cells) can be reproduced by transplanting WB1 cells into the diabetic mice. ${ }^{9,10}$ Based on these observations, we come to the conclusion that expression of both Pdx1-VP16 and Pax4 can initiate and facilitate the transdifferentiation of the hepatic stem-like WB cells into glucose-responsive liverderived functional insulin-producing cells.

We stress that WB-1A cells are genetically modified liver-derived insulin-producing cells, not true $\beta$-cells. These cells were inappropriately regulated, as demonstrated by both in vitro glucose-stimulated insulin release, as well as in vivo animal studies. In particular, extended implantation by WB-1A cells in mice led to apparently fatal hypoglycemia. Therefore, at this time and in this setting, we do not believe WB-1A cells should be compared to fully functional islets with respect to glucose regulation. Although we are fully aware of that our liver-derived insulin-producing cells are not true pancreatic $\beta$-cells, we are making progress toward the goal of understanding how cellular gene expression may be manipulated to generate liver-derived insulin-producing cells having similar, if not identical, characteristics to those of pancreatic islets for future attempts at cell replacement therapy.

The major goal for cell replacement therapy is to generate glucose-regulated insulin-producing cells. Although co-expression of both $P d \times 1-V P 16$ and Pax4 can transdifferentiate hepatic WB cells into glucose-responsive cells, as noted our long-term animal studies showed persistent hypoglycemia occurring in WB-1A transplanted mice. As Pax4 expression is driven by the strong constitutive EF1 $\alpha$ promoter in WB-1A cells, the significant hypoglycemic state in the transplanted mice failed to taper off or to shut down Pax4 expression, even though the immediate upstream $N k x 2.2$ gene was markedly downregulated by the persistent expression of Pax4. An alternative approach, therefore, might be to control Pax4 expression with a liver-specific promoter such as albumin that will shut down the exogenous Pax4 expression upon complete hepatic cell conversion into pancreatic cells ${ }^{5}$ or transient 
Pax4 expression. In contrast, $P d x 1-V P 16$ or $\mathrm{Pdx} 1$ expression alone reprograms hepatic cells toward the pancreatic endocrine differentiation pathway and generates precursors of insulin producing cells. ${ }^{9,21}$ When such cells were transplanted into diabetic animals, euglycemia was maintained until the end of the observation period (ie, 4 months) without animal loss. Several important conclusions can be derived from the aforementioned observations. First, hepatic WB cells should be reprogrammed from a higher position (eg, Pdx1) in the cascade of $\beta$-cell transcription activation, leaving the many downstream steps to be regulated by changes in the blood glucose levels. Second, it is possible that WB-1 cells are in a stem-like stage in some respects resembling $\beta$-cell precursors, with some cells remaining at this stage being available to replenish the mature form of $\beta$-like cells on an asneeded basis in response to changes of the blood glucose levels. Third, it may be more practical to employ a combination of early and late-stage $\beta$-cell transcription factor to transdifferentiate the liver cells; and the late-stage transcription factor should be controlled by either a glucose-regulated or a liverspecific promoter.

Overall, our comparative analysis of $P d x 1-V P 16$ expressing WB-1 cells and Pdx1-VP16/Pax4-expressing WB-1A cells suggests that Pax4 expression in WB-1A cells facilitates Pdx1-VP16 to reprogram liver stem cells into functional $\beta$-cell-like phenotype. These cells respond both to a high-glucose challenge in vitro and to hyperglycemia in vivo by releasing insulin. However, they are apparently unable to respond to the lower blood glucose levels in the NOD-scid mice by reducing insulin release, due to the constitutive expression of Pax4. Therefore, a more fruitful approach for transdifferentiating liver cells into pancreatic $\beta$-like insulin-producing cells may be to select suitable gene(s) from a higher position in the cascade controlling transcription during $\beta$-cell development and/or suitable glucoseregulated or liver-specific promoters. This selection might fulfill the requirement for selective hepatic cell transdifferentiation into pancreatic $\beta$-like insulin-producing cells, while, at the same time, allowing for the genetically engineered $\beta$-like cells to respond effectively to both upward and downward changes in blood glucose levels.

\section{Acknowledgements}

We thank Christopher E Wright for the anti-Pdx1 antibody, Christopher B Newgard for rat INS-1 cell line (clone 832/13), Michael German for Pax4 cDNA, and Marko E Horb for Pdx1-VP16 plasmid. We thank Yu-Ping Sun, for technical support. National Institutes of Health research grants DK064054 and DK071831 to Li-Jun Yang and NIH/T32 AR007603Institutional Postdoctoral Fellowship to Dong-Qi Tang supported this research project.

\section{References}

1 Deutsch G, Jung J, Zheng M, et al. A bipotential precursor population for pancreas and liver within the embryonic endoderm. Development 2001;128: 871-881.

2 Rao MS, Reddy JK. Hepatic transdifferentiation in the pancreas. Semin Cell Biol 1995;6:151-156.

3 Rao MS, Bendayan M, Kimbrough RD, et al. Characterization of pancreatic-type tissue in the liver of rat induced by polychlorinated biphenyls. J Histochem Cytochem 1986;34:197-201.

4 Tosh D, Slack JM. How cells change their phenotype. Nat Rev Mol Cell Biol 2002;3:187-194.

5 Horb ME, Shen CN, Tosh D, et al. Experimental conversion of liver to pancreas. Curr Biol 2003;13: 105-115.

6 Ferber S, Halkin A, Cohen $\mathrm{H}$, et al. Pancreatic and duodenal homeobox gene 1 induces expression of insulin genes in liver and ameliorates streptozotocininduced hyperglycemia. Nat Med 2000;6:568-572.

7 Ber I, Shternhall K, Perl S, et al. Functional, persistent, and extended liver to pancreas transdifferentiation. J Biol Chem 2003;278:31950-31957.

8 Kojima H, Fujimiya M, Matsumura K, et al. NeuroDbetacellulin gene therapy induces islet neogenesis in the liver and reverses diabetes in mice. Nat Med 2003;9:596-603.

9 Cao LZ, Tang DQ, Horb ME, et al. High glucose is necessary for complete maturation of pdx-1-vp16expressing hepatic cells into functional insulin-producing cells. Diabetes 2004;53:3168-3178.

10 Wilson ME, Scheel D, German MS. Gene expression cascades in pancreatic development. Mech Dev 2003; 120:65-80.

11 Sosa-Pineda B, Chowdhury K, Torres $\mathrm{M}$, et al. The Pax4 gene is essential for differentiation of insulinproducing beta cells in the mammalian pancreas. Nature 1997;386:399-402.

12 Gittes GK, Rutter WJ. Onset of cell-specific gene expression in the developing mouse pancreas. Proc Natl Acad Sci USA 1992;89:1128-1132.

13 Brun T, Franklin I, St Onge L, et al. The diabeteslinked transcription factor PAX4 promotes \{beta\}-cell proliferation and survival in rat and human islets. J Cell Biol 2004;167:1123-1135.

14 Smith SB, Ee HC, Conners JR, et al. Paired-homeodomain transcription factor PAX4 acts as a transcriptional repressor in early pancreatic development. Mol Cell Biol 1999;19:8272-8280.

15 Chang LJ, Zaiss AK. Lentiviral vectors. Preparation and use. Methods Mol Med 2002;69:303-318.

16 Chang LJ, Zaiss AK. Self-inactivating lentiviral vectors and a sensitive Cre-loxP reporter system. Methods Mol Med 2003;76:367-382.

17 Grisham JW, Coleman WB, Smith GJ. Isolation, culture, and transplantation of rat hepatocytic precursor (stem-like) cells. Proc Soc Exp Biol Med 1993;204: 270-279.

18 Couchie $\mathrm{D}$, Holic $\mathrm{N}$, Chobert $\mathrm{MN}$, et al. In vitro differentiation of WB-F344 rat liver epithelial cells into the biliary lineage. Differentiation 2002;69: 209-215.

19 Hohmeier HE, Mulder H, Chen G, et al. Isolation of INS-1-derived cell lines with robust ATP-sensitive $\mathrm{K}+$ channel-dependent and -independent glucose-stimulated insulin secretion. Diabetes 2000;49:424-430. 
20 Yang $\mathrm{L}$, Li S, Hatch $\mathrm{H}$, et al. In vitro trans-differentiation of adult hepatic stem cells into pancreatic endocrine hormone-producing cells. Proc Natl Acad Sci USA 2002;99:8078-8083.

21 Tang DQ, Lu S, Sun YP, et al. Reprogramming liverstem WB cells into functional insulin-producing cells by persistent expression of Pdx1- and Pdx1-VP16 mediated by lentiviral vectors. Lab Invest 2006;86:83-93.

22 Tang DQ, Cao LZ, Burkhardt BR, et al. In vivo and in vitro characterization of insulin-producing cells obtained from murine bone marrow. Diabetes 2004;53: 1721-1732.

23 Macfarlane WM, McKinnon CM, Felton-Edkins ZA, et al. Glucose stimulates translocation of the homeodomain transcription factor PDX1 from the cytoplasm to the nucleus in pancreatic beta-cells. J Biol Chem 1999;274:1011-1016.

24 Wang J, Elghazi L, Parker SE, et al. The concerted activities of Pax4 and Nkx2.2 are essential to initiate pancreatic beta-cell differentiation. Dev Biol 2004;266: 178-189.

25 Blyszczuk P, Czyz J, Kania G, et al. Expression of Pax4 in embryonic stem cells promotes differentiation of nestin-positive progenitor and insulin-producing cells. Proc Natl Acad Sci USA 2003;100:998-1003.

26 Teitelman G, Alpert S, Polak JM, et al. Precursor cells of mouse endocrine pancreas coexpress insulin, glucagon and the neuronal proteins tyrosine hydroxylase and neuropeptide Y, but not pancreatic polypeptide. Development 1993;118:1031-1039.

Supplementary Information accompanies the paper on the Laboratory Investigation website (http:// www.nature.com/labinvest) 